\title{
Extraction of Stationary Spectral Components Using Stochastic Variability
}

\author{
David Cárdenas-Peña, \\ Juan David Martínez-Vargas, and Germán Castellanos-Dominguez \\ Signal Processing and Recognition Group, Universidad Nacional de Colombia, \\ Km. 9, Vía al aeropuerto, Campus la Nubia, Caldas, Manizales, Colombia \\ \{dcardenasp, jmartinezv, cgcastellanosd\}@unal.edu.co
}

\begin{abstract}
Biosignal recordings are widely used in the medical environment to support the evaluation and the diagnosis of pathologies. Nevertheless, the main difficulty lies in the non-stationary behavior of the biosignals, making difficult the obtention of patterns characterizing the changes in physiological or pathological states. Thus, the obtention of the stationary and non-stationary components of a biosignal poses still an open issue. This work proposes a methodology to detect time-homogeneities based on time-frequency analysis aiming to extract the non-stationary behavior of the biosignal. Two homogeneity constraints are introduced as the measure of stochastic variability of the considered dataset. The first one is the relevance value, which gives information about the contribution of the spectral component to the process. The second one is based on the first and second moments of stochastic variability map, being related to the uniformity along the time of each spectral component. Results show an increase in the stationarity of the reconstructions from the enhanced time-frequency representations. Moreover, the inter class distance for the reconstructed time-series show more discrimination on the stationary component than on the non-stationary one. Those extracted components tend to meet the requirement of most algorithms proposed for other tasks, such as biosignal classification problems, leading to a performance increase of the methodologies.
\end{abstract}

Keywords: Time-evolving Latent Variable Decomposition, Multivariate Locally Stationary Time Series, Stationarity Enhancement.

\section{Introduction}

In biosignal applications, it is often of interest to be able to separate an observed time series into two or more groups with different stochastic behavior [4]. In particular, there is a need for distinguishing stationary from non-stationary components, either because its assumption is a pre-requisite for applying most of standard algorithms devoted to steady-state regimes, or because its breakdown conveys specific information in evolutive contexts, as remarked in [8]1. In the analysis of biomedical data, corresponding recordings usually appear nonstationary (temporal and spectral characteristics of the signal vary over time), 
although there exist stationary sources, these are not discernible, since superpositions of stationary and non-stationary components can be measured.

Commonly, the time domain non-stationariness can be quantitative measured by the variation of either first two statistical moments over time (second order process). Instead, the non-stationary upon the spectrum domain of biological signals has been emphasized more recently, because of the corresponding physiological meanings of some spectral components [3], for instance, for Heat Rate Variability (HRV) recordings, the spectral components can provide information about the relative inputs of the two autonomic nervous system mutually antagonistic components the sympathetic one, which acts to increase heart rate, and the parasympathetic component acting to slow the heart and to dilate blood vessels [4]. Nevertheless, even the traditional time-averaged spectrum of nonstationary signals, being widely used, is not appropriate for quantifying the temporal variability in the spectrum. Therefore, techniques devoted to provide clear images of the energy distribution on the time-frequency $(t-f)$ plane, directly demonstrating the non-stationarity of the signal, have been proposed for decomposition into stationary and non-stationary components, namely, $t-f$ localized linear splines, $t-f$ representations, wavelets, empirical mode decomposition, and stationary subspace analysis [7. Generally, extraction of stationary components from real-valued biosignal can be provided by using the following two subsequent stages: $i$ ) decomposition of underlying time series, by properly handling the signal model within a stochastic subspace framework, and $i i$ ) searching of the needed number of components to match a priori given stationary homogeneity constrains.

This work is based on evolutionary time-frequency $(t-f)$ analysis, for which testing of the time-homogeneity constraints of the evolutionary spectra is evaluated at different instants of time by using multivariate time series subspace representation. In this sense, a new methodology for stationarity enhancement on biosignals is introduced. The paper is organized as follows: The Section 2 is dedicated to the description of the methods required to perform the stationarity enhancement; in Section [3, the experimental setup is described and obtained results are presented. Finally, the results are properly discussed in Section 4.

\section{Background}

\subsection{Enhanced $t$ - $f$ Representation}

Decomposition into stationary and non-stationary components is carried out upon enhanced $t$-f representation of the input data. In particular, the short time Fourier transform is employed introducing a time localization concept by means of a tapering window function of short duration, $\phi$, that is going along the underlying biosignal, $y(t)$, as follows:

$$
\boldsymbol{S}_{y}(t, f)=\left|\int_{T} y(\tau) \phi(\tau-t) e^{-j 2 \pi f \tau} d \tau\right|^{2}, \boldsymbol{S}_{y}(t, f) \in \mathbb{R}^{+}
$$


with $t, \tau \in T, f \in F$. Based on introduced spectrogram of Eq. (11), the corresponding $t-f$ representation matrix, $\boldsymbol{S}_{y} \in \mathbb{R}^{T \times F}$, can be described by the row vector set, $\boldsymbol{S}_{y}=\left[\boldsymbol{s}_{1} \ldots \boldsymbol{s}_{f} \ldots \boldsymbol{s}_{F}\right]^{\top}$, with $\boldsymbol{s}_{f} \in \mathbb{R}^{1 \times T}$, where the vector $\boldsymbol{s}_{f}=[s(f, 1) \ldots s(f, t) \ldots(f, T)]$, with $s(f, t) \in \mathbb{R}$, is each one of the time-variant spectral decomposition components at frequency $f$, and equally sampled through the time axis.

\subsection{Measure of Stochastic Variability}

Several variability analysis techniques suitable for clinical applications had been proposed, including statistical, geometric, energetic, informational, and invariant measures [2]. In this research, the amount of stochastic variability of the spectral component set is computed following the approach given in [4, that is based on time-variant decomposition estimated by adapting in time any of commonly used latent variable techniques, upon which a piecewise stationary restriction is imposed [6]. So, under the locally stationary assumption, consistent estimates of the time-varying spectral density matrix are obtained and consequently consistent estimates of the time-varying eigenvalues and eigenvectors may be accomplished [5]. Namely, the time-evolving principal component analysis is extended to the dynamic feature modeling by stacking the input observation matrix in the following manner:

$$
\boldsymbol{\Xi}_{y}=\left[\begin{array}{cccc}
\boldsymbol{s}_{1}^{1} & \boldsymbol{s}_{2}^{1} & \cdots & \boldsymbol{s}_{F}^{1} \\
\boldsymbol{s}_{1}^{2} & \boldsymbol{s}_{2}^{2} & \cdots & \boldsymbol{s}_{F}^{2} \\
\vdots & \vdots & \vdots & \vdots \\
\boldsymbol{s}_{1}^{M} & \boldsymbol{s}_{2}^{M} & \cdots & \boldsymbol{s}_{F}^{M}
\end{array}\right], \boldsymbol{\Xi}_{y} \in \mathbb{R}^{M \times F T}
$$

where vector $\boldsymbol{s}_{f}^{i}$ corresponds to $f$-th short-term spectral component estimated from the $i$-th spectrogram matrix, $\boldsymbol{S}_{y}^{i}$, which is related to the $i$-th object, with $i \in$ $M$. Accordingly, the amount of stochastic variability of the spectral component set is computed by the singular value decomposition calculation over observation matrix in Eq. (2). So, the following time-variant relevance measure is carried out [6]:

$$
\boldsymbol{g}\left(\boldsymbol{\Xi}_{y} ; \tau\right)=[\chi(1) \cdots \chi(\tau) \cdots \chi(F T)]^{\top}, \in \mathbb{R}^{F T \times 1}
$$

being $\chi(\tau)=\boldsymbol{E}\left\{\left|\lambda_{f}^{2} v_{f}(\tau)\right|: \forall f \in F\right\}$, where $\left\{\lambda_{f}\right\}$ is the relevance eigenvalue set of matrix $\boldsymbol{\Xi}_{y}$, and scalar-valued $v_{f}(\tau)$ is the respective element at $\tau$ moment, with $\tau=1, \ldots, F T$ that indexes every one of the relevance values computed for the whole time-variant data set (notation $\boldsymbol{E}\{\cdot\}$ stands for the expectation operator). At the end of the multivariate procedure, a relevance map $\Gamma(t, f)=$ $\left[g\left(s_{1}\right) \ldots g\left(s_{f}\right) \ldots g\left(s_{F}\right)\right], \in \mathbb{R}^{T \times F}$ is achieved that contains stochastic variability measured for the whole spectral component set, $\left\{\boldsymbol{s}_{f}\right\}$.

Matching stochastic homogeneity constrains: From the relevance map $\Gamma(t, f)$, the amount of information given for each spectral component, $\boldsymbol{s}_{f}$, can be derived 
by averaging the relevance matrix over the frequency axis, yielding the relevance marginal $\gamma(f)$. Thus, the higher the value of $\gamma(f)$, the more relevant the spectral component $\boldsymbol{s}_{f}$. With this constrains in mind, a weighting $t-f$ function can be built with those frequency components exceeding a relevance threshold $\eta$, as follows:

$$
\boldsymbol{\omega}_{f}(\eta)=\left\{\begin{array}{ll}
1, & \text { if } \gamma(\boldsymbol{f})>\boldsymbol{\eta} \\
0, & \text { otherwise }
\end{array}, \quad \forall f \in F .\right.
$$

Additionally, taking each relevant spectral component as a time-series $\boldsymbol{s}_{f}$, regarded as stochastic process, any randomness structure estimator must remain constant in time, by instance, the mean value. This work proposes to take the following assumption:

$$
\left\|\boldsymbol{E}\left\{g\left(\boldsymbol{s}_{f}\right) ; t\right\}-\boldsymbol{E}\left\{g\left(\boldsymbol{s}_{f}\right) ; t-\tau\right\}\right\| \leq \varepsilon, \quad \forall t, \tau \in T
$$

with $\varepsilon \rightarrow 0$. Thus, the weighting $t-f$ function can be redefined with those relevant frequency bands satisfying the previous conditions, becoming in a $\eta, \varepsilon$ dependant function, $\boldsymbol{\omega}_{f}(\eta, \varepsilon)$.

In practice, the extraction of non-stationary components from a random signal formally can be related as filtration task, carried out under the following assumptions [7]:

- the observed signals are linear superpositions of stationary and non-stationary sources, so, an observable given time series vector, $\boldsymbol{y}$, is separated into two unobservable components, i.e., a stationary and non-stationary, respectively;

$$
\boldsymbol{y}=\boldsymbol{y}^{*}+\varsigma,
$$

- the non-stationarity component, $\boldsymbol{\varsigma}$, is a second order measurable stochastic process.

Hence, the main assumption in this research is that by selecting the relevant and stationary frequency bands of the $t-f$ maps, according with the level of stochastic homogeneity of the random variable $\boldsymbol{s}_{f}$, a given time series $\boldsymbol{y}$ can be split into the stationary $\boldsymbol{y}^{*}$ and non-stationary $\boldsymbol{\varsigma}$ parts.

Measuring the stationarity degree of the time series: As proposed in [1], firstly, a set of $J$ stationary surrogate signals $\left\{s_{j}: j=1, \ldots, J\right\}$ is computed from a given signal. Then, a contrast measure, for a given time series $y$, is defined as:

$$
c_{n}(y):=d\left(\boldsymbol{S}_{y}(t, f), \boldsymbol{E}\left\{\boldsymbol{S}_{y}(t, f): \forall t \in T\right\}\right)
$$

where $d \in \mathbb{R}^{+}$is some suitable spectral distance, in this case the Kullback-Leibler distance was used. Finally, the index of non-stationarity is determined as:

$$
\kappa=\sqrt{\frac{\operatorname{var}\left(c_{n}(y): \forall t \in T\right)}{\boldsymbol{E}\left\{\operatorname{var}\left(c_{n}\left(s_{j}\right): \forall t \in T\right): \forall j\right\}}}
$$




\section{Experimental Set-up}

\subsection{Database Description}

The database holds a available collection of 1-min HRV segments selected from Physionet, which holds 70 electrocardiographic recordings, each one including a set of reference annotations added every minute of the recording indicating either the presence of absence of apnoea during each segment. Finally, 600 HRV segments of 1-minute length (300 apneic and 300 normal labeled) were selected from 25 training recordings to build the dataset [4].

\subsection{Splitting into Stationary and Non-stationary Subspaces}

Time-Frequency Representations Enhancement of Estimated Time Series: In this work, according to the spectral HRV signal properties, the STFT based quadratic spectrogram is computed by sliding windows for the following set of estimation parameters: a $32.5 \mathrm{~ms}$ hamming window, $50 \%$ of overlapping, and 512 frequency bins within a range of 0 to $1 \mathrm{~Hz}$. For the sake of medical interpretation, the whole frequency rank is splitted into sub-frequency bands, termed Low Frequency (LF) spectral band $(\boldsymbol{f} \in[0.04-0.15] H z)$ and High Frequency (HF) spectral band $(\boldsymbol{f} \in[0.15-0.5] H z)$.

Weighting Function Selection: The main core of this approach is to find the stationary and non-stationary components of a given time series. For this aim, the weighting $t-f$ function derived from the relevance map, must be tuned to optimize the level of stationarity of the time-series, by assuming: $(i)$ the higher the relevance threshold $\eta$, the more relevant the selected spectral components $\boldsymbol{s}_{f}^{*}$; and $(i i)$ the larger value of $\varepsilon$, the more presence of non-stationary components to the signal. Thus, once the thresholds $\eta$ and $\varepsilon$ are selected, the stationary $\boldsymbol{y}^{*}$ and non-stationary $\varsigma$ parts of the time series can be derived by the inverse STFT.

To test the performance of the proposed methodology, two measures are stablished. Firstly, the $\kappa$ index assessing the degree of signal non-stationarity is used. So, the $\kappa$ values are computed for each recording on the dataset $\left(\kappa_{\boldsymbol{y}}\right)$ and its stationary $\left(\kappa_{\boldsymbol{y}^{*}}\right)$ and non-stationary $\left(\kappa_{\boldsymbol{\varsigma}}\right)$ reconstructions, obtained with the proposed approach. Consequently, the lower the $\kappa$ index, the more stationary the time-series.

Secondly, intra and inter class distance functions are employed to measure quantitatively the influence of the stationarity and non-stationarity on the groupping of the signals. A Hausdorff distance scheme was employed as the distance between two sets (classes). Since such scheme requires the comparison of all possible pairs of time-variant subjects, the correlation distance was choosen as the pairwise subject comparison. 
Since the relevance map is computed for both spectral bands, $\eta$ and $\varepsilon$ have to be estimated for each of them. The marginals $\gamma_{L F}(f)$ and $\gamma_{H F}(f)$ are estimated for their respective bands as follows:

$$
\begin{aligned}
\gamma_{L F}(f) & =\boldsymbol{E}\left\{g\left(\boldsymbol{s}_{f}\right) ; t\right\} ; \forall f \in[0.04-0.15] \mathrm{Hz} \\
\gamma_{H F}(f) & =\boldsymbol{E}\left\{g\left(\boldsymbol{s}_{f}\right) ; t\right\} ; \forall f \in[0.15-0.5] H z,
\end{aligned}
$$

while the ratios $\varepsilon_{L F}(f)$ and $\varepsilon_{H F}(f)$ are defined as, $\varepsilon(f)=\boldsymbol{\sigma}(f) / \gamma(f)$, being $\boldsymbol{\sigma}(f)$ the standard deviation of $g\left(\boldsymbol{s}_{f}\right)$. Finally, the marginals $\gamma(f)$ and ratios $\varepsilon(f)$ are normalized in the interval $[0,1]$.

\section{Results and Discussion}

Figure 1(a) shows the results of $k$ for the iterative procedure, while varying the relevance threshold $\eta$ between $[0.1,0.8]$ with steps of 0.1 , and the stationarity threshold $\varepsilon$ between $[0.1,0.7]$ with steps of 0.1 . As expected, the higher values of $k\left(\kappa_{\varsigma}>\kappa_{y^{*}}\right)$, are presented for lower values of $\varepsilon$. In that case, the variability of the spectral components is highly restricted, therefore the presence of nonstationary components is low.

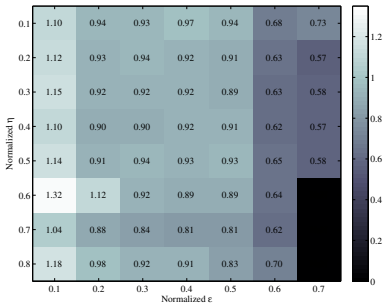

(a) Stationarity measure

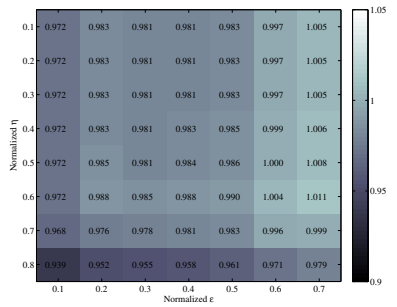

(b) Inter class distancefor $\boldsymbol{y}^{*}$

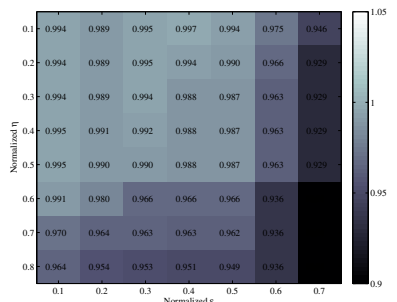

(c) Inter class distance for $\varsigma$

Fig. 1. Experimental results for tested configurations of $\varepsilon$ and $\eta$

Figures 1(b) and 1(c) depict the distance measure between apneic and normal classes for both reconstructions, stationary and non-stationary, respectively. Two facts have to be highlighted in those results. Firstly, the larger the $\varepsilon$ threshold, the larger the inter-class distance for the stationary reconstruction $\left(\boldsymbol{y}^{*}\right)$ and the shorter the inter-class distance for the non-stationary reconstruction ( $\varsigma$ ). Which means that the stationary components of such biosignals contain the inner class dynamics. Secondly, it is expected to obtain higher distances on lower values of the $\eta$ threshold. Such behavior is related to the number of relevant components used on the reconstructions. Since higher thresholds turn off more components on each class, all subjects are equally represented on such frequencies, reducing the pairwise correlation distance of the subjects.

Figure 2 2 shows an example of the estimated $t$ - $f$ representation (left), along to the obtained relevance map for both spectral bands (center) and the relevance 
marginal $\gamma(f)$, as well as its standard deviation $\boldsymbol{\sigma}(f)$ (right). Moreover, as an example, the frequency weighting function for $\eta=\varepsilon=0.6$ is plotted as a blue line over the marginal. It can be seen that the proposed measure attempts to select not only the more relevant but also the more steady components of the map. Frequencies with higher relevance are allowed to have higher variance and the less relevant frequencies require a more steady behavior to be taken into account.
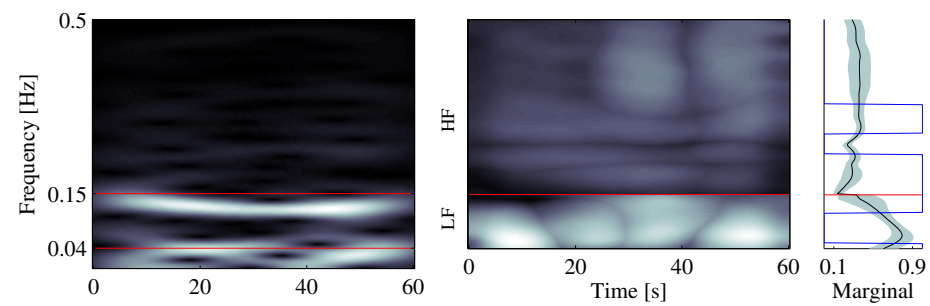

Fig. 2. Relevance map and relevance marginal estimation for each spectral band of the HRV database. Frequency weighting function in blue.

An example of the reconstruction procedure is shown in Figure 3 In the left side, a common pathological signal is depicted along with its $t$ - $f$ representation; the relevant stationary and relevant non-stationary component of the $t$ - $f$ enhanced by the weighting function are presented in the middle top and middle bottom, respectively; in the right side are shown the reconstructions of each part of the signal. It must be highlighted that the stationary reconstruction is smoother than the non-stationary side. This kind of signals tends to meet the requirements of most of the standard algorithms devoted to steady-state regimes.
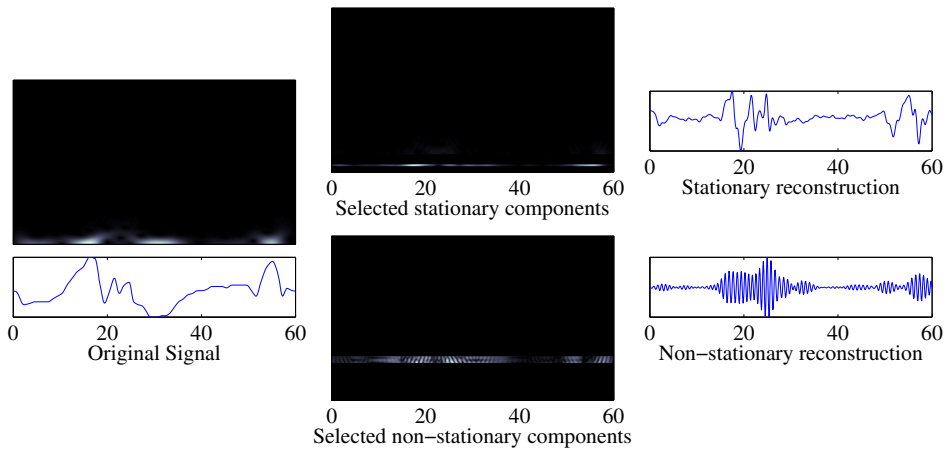

Fig. 3. Reconstruction procedure for both stationary and non-stationary components of the signal 


\section{Conclusions}

A new methodology for stationarity enhancement on biosignals is presented. The performance is tested on a well known database, which comprise non-stationary behaviors, namely, heart rate variability recordings. The methodology estimates a spectral weighting function from a stochastic variability map. Such function is applied as a filter enhancing the stationary components of the signal. As measure of the non-stationarity the $\kappa$, proposed by [1] is used. Results show an increase in the stationarity of the reconstructions from the enhanced $t-f$ representations as well as an improvement on the representation of each class As future work, the use of several non-stationarity measures is proposed, as well as the use of the methodology for solving non-stationary signal discrimination issues.

Acknowledgments. This work is supported by "Centro de Investigación e Innovaciónn de Excelencia - ARTICA, and Programa Nacional de Formación de Investigadores "Generación del Bicentenario", 2011, financed by COLCIENCIAS".

\section{References}

1. Borgnat, P., Flandrin, P., Honeine, P., Richard, C., Xiao, J.: Testing stationarity with surrogates: A time-frequency approach. Submitted to IEEE Trans. Signal Processing 58(7), 3459-3470 (2009)

2. Bravi, A., Longtin, A., Seely, A.: Review and classification of variability analysis techniques with clinical applications. BioMedical Engineering OnLine 10(1), 90 (2011)

3. Cao, C., Slobounov, S.: Application of a novel measure of eeg non-stationarity as shannon- entropy of the peak frequency shifting for detecting residual abnormalities in concussed individuals. Clinical Neurophysiology 122(7), 1314-1321 (2011)

4. Martinez-Vargas, J.D., Sepulveda-Cano, L.M., Travieso-Gonzalez, C., CastellanosDominguez, G.: Detection of obstructive sleep apnoea using dynamic filter-banked features. Expert Systems with Applications 39(10), 9118-9128 (2012)

5. Ombao, H., Ringo Ho, M.: Time-dependent frequency domain principal components analysis of multichannel non-stationary signals. Comput. Stat. Data Anal. 50(9), 2339-2360 (2006)

6. Sepulveda-Cano, L., Acosta-Medina, C.: Castellanos-Dominguez G. Relevance Analysis of Stochastic Biosignals for Identification of Pathologies. EURASIP Journal on Advances in Signal Processing 2011, 10 (2011)

7. Von Buenau, P., Meinecke, F., Kiraly, F., Mueller, K.: Finding stationary subspaces in multivariate time series. Physical Review Letters 103(21), 214101-1-214101-4 (2009)

8. Weng, X., Shen, J.: Finding discordant subsequence in multivariate time series. In: 2007 IEEE International Conference on Automation and Logistics, pp. 1731-1735 (August 2007) 